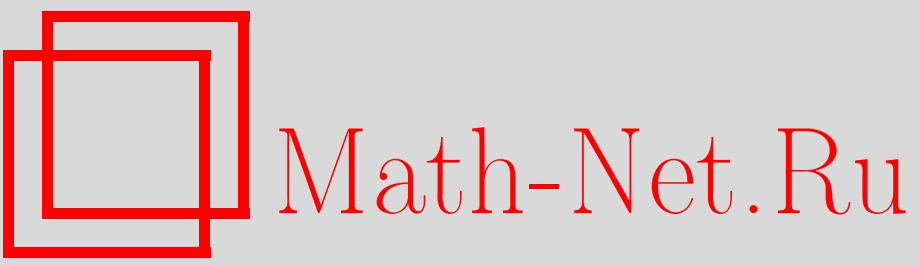

С. С. Рышков, Е. А. Большакова, К теории коренных параллелоэдров, Изв. РАН. Сер. матем., 2005, том 69, выпуск 6, 187-210

DOI: https://doi.org/10.4213/im672

Использование Общероссийского математического портала Math-Net.Ru подразумевает, что вы прочитали и согласны с пользовательским соглашением

http://www . mathnet.ru/rus/agreement

Параметры загрузки:

IP: 54.162 .127 .20

26 апреля 2023 г., 14:34:22 
УДК $511.9+514.174$

\author{
С. С. Рышков, Е. А. Большакова
}

\title{
К теории коренных параллелоэдров
}

В 1998 г. первый автор анонсировал теорему о представлении с точностью до аффинного преобразования каждого примитивного $n$-мерного параллелоэдра в виде взвешенной суммы Минковского нескольких из конечного числа специально расположенных $n^{\prime}$-мерных, $n^{\prime} \leqslant n$, коренны $x^{1}$ параллелоэдров. В статье приведено подробное доказательство этой теоремы в уточненном и максимально расширенном виде.

Библиография: 16 наименований.

\section{§1. Введение}

1.1. Соглашения, сокращения и обозначения. В настояшей статье приняты следующие обозначения:

- ПКФ - положительно определенная квадратичная форма;

- $n \Pi$ П $\Phi-\Pi К \Phi$ от $n$ переменных;

- $\oplus$ - векторное сложение (суммирование по Минковскому), обычно с центром в начале координат;

- $\|A\|=\left(\operatorname{tr} A A^{T}\right)^{1 / 2}=\left(\operatorname{Sp} A A^{T}\right)^{1 / 2}$ - норма матрицы $A ;$

- $E^{n}-n$-мерное евклидово пространство, заданное ортонормированным базисом $\mathcal{E}$ с началом координат $O$;

- $E^{k} \subset E^{n}$ - координатное подпространство, отвечающее первым $k$ координатам;

- $Z^{n}$ - множество целочисленных строк $\left(x_{1}, x_{2}, \ldots, x_{n}\right)$;

- $f(x)=\sum_{i, j=1}^{n} a_{i j} x_{i} x_{j}=\sum \sum a_{i j} x_{i} x_{j}$ - квадратичная форма (как правило, положительно определенная) от $n$ переменных и $\left(a_{i j}\right)=A=A^{T}$ - ее матрица;

- $\mathbb{E}^{N}$, где $N=\frac{n(n+1)}{2},-$ пространство коэффициентов квадратичных форм с началом в точке $\mathcal{O}$;

$-\mathbb{K}^{N} \subset \mathbb{E}^{N}$ - конус положительности;

- $(x, y)=\sum_{i=1}^{n} x_{i} y_{i}-$ скалярное произведение арифметических векторов $x$ и $y$.

\footnotetext{
${ }^{1}$ Коренной- к корню в различ. знач. относящийся, но более в знач. переносном: основной, начальный, настоящий, первобытный (В. Даль).

Работа выполнена при финансовой поддержке РФФИ (грант № 05-01-00170, выданный первому автору) и INTAS (грант № 03-51-5070, выданный обоим авторам).
} 
Для удобства будем использовать также и матричную запись. Векторы заданы строками своих координат. Таким образом,

$$
(x, y)=x y^{T}, \quad f(x)=x A x^{T}, \quad f(x, y)=x A y^{T} .
$$

Рассматриваемые в статье "разбиения" евклидова пространства на выпуклые замкнутые многогранники - это "классические разбиения", т. е. такие покрытия пространства многогранниками, элементы которых либо не пересекаются, либо пересекаются только по границе.

Запись вида $i=\overline{1, \sigma}$ означает, что индекс $i$ принимает значения $1,2, \ldots, \sigma$.

Для того чтобы настоящая статья была в какой-то мере самодостаточной, а также для того чтобы избежать разночтений в терминологии, мы, в нужной нам форме, приводим здесь многие факты из весьма трудной для чтения и библиографически достаточно редкой работы Г. Ф. Вороного [1].

1.2. Предмет статьи. Предметом изучения настоящей статьи являются параллелоэдры. Кратко напомним основные понятия и факты, подробное описание которых можно найти, например, в [1]-[5].

Пусть каждый элемент какого-либо разбиения $n$-мерного евклидова пространства на ограниченные вьпуклые многогранники параллельно конгруэнтен одному и тому же многограннику. Такие и только такие многогранники называются $n a$ раллелоэдрами. Если рассматриваемое разбиение есть разбиение грань-в-грань, то соответствующий параллелоэдр часто называют нормальныц.м. Если разбиение и примитивно, т. е. если в каждой его вершине сходится ровно $n+1$ элемент разбиения, то параллелоэдр называется примитивныцм. Отметим, что каждый примитивный параллелоэдр есть примитивный многогранник (т. е. в каждой вершине сходятся ровно $n$ граней), однако не каждый примитивный многогранник, являюшийся параллелоэдром, есть примитивный параллелоэдр (пример - куб).

Одна из основных теорем теории параллелоэдров - теорема Г. $\Phi$. Вороного [1] состоит в том, что каждый примитивный параллелоэдр есть аффинный образ области Дирихле-Вороного, отвечающей некоторой ПКФ. Единственность такой ПКФ доказана в [6]. Теорема Вороного распространена Б. Н. Делоне [7] на все параллелоэдры, имеюшие размерность $n \leqslant 4$.

Примитивные параллелоэдры классифицируются по типам (подробнее об этом см. $\S 2)$, однако связь между параллелоэдрами одного и того же типа до последнего времени была раскрыта далеко не полностью.

В заметках [8]-[10] первый автор настоящей работы анонсировал (с последовательными уточнениями) теорему о том, что любой $n$-мерный $(n>0)$ примитивный параллелоэдр представляется в виде взвешенной суммы Минковского параллелоэдров из некоторого, конечного для всякого $n$, набора параллелоэдров различных размерностей $k \leqslant n$. Эти параллелоэдры автор назвал коренны.ми.

Таким образом, изучение всего многообразия примитивных параллелоэдров было сведено к изучению коренных параллелоэдров.

Коренные параллелоэдры (из-за трудности адекватного перевода этого слова на английский язык) в некоторых из предыдущих публикаций мы называли, кроме того, базисными, что в достаточной мере отражает их сушность. 
В заметке [8] было указано, что доказательство теоремы о коренных параллелоэдрах легко получается применением результатов из $\S 69-76,85$ и 86 работы [1]. Такая ссылка уже давала понять читателю, что доказательство теоремы имеет не только геометрический, но и аналитический характер. В связи с непониманием некоторыми читателями этого обстоятельства в работе [11] были приведены аналитическая формулировка теоремы и дополнительные разъяснения.

Первоначальное доказательство базировалось на идее перенесения $L$-структуры примитивных параллелоэдров некоторого (арифметического) типа на границу области этого типа. Однако подробное изложение доказательства оказалось технически достаточно трудным, поэтому авторы, уже совместно, отыскали другое доказательство, как им кажется, гораздо более простое. При этом теорема была распространена на области Дирихле-Вороного, отвечающие произвольным ПКФ. Следует отметить большой вклад, внесенный вторым автором в доказательства теоремы 4.1 и леммы 3.6 (о "трубочке").

Здесь уместно вспомнить, что, как неоднократно рассказывал Б. Н. Делоне, Г. Ф. Вороной мыслил очень "геометрически", и только давление внешних обстоятельств в лице "академика профессора Маркова" (см. [12]) заставило его формулировать и доказывать свои результаты в аналитической форме. В дальнейшем многие понятия и результаты из работ Вороного были блестяще сформулированы Б. Н. Делоне [13], [3] геометрически, и именно в этой форме приобрели известность и популярность. О соотношении упомянутых определений, а также о понятиях арифметического и геометрического $L$-типов см. [14].

1.3. Формулировка основных теорем. Далее параллелоэдр понимается как область Дирихле-Вороного для некоторой ПКФ согласно первоначальному определению такой области в работе [1].

ОПРЕДЕЛЕнИЕ 1.1. Множество $\Sigma$ параллелоэдров называется системой коренных параллелоэдров тогда и только тогда, когда оно удовлетворяет следуюшим условиям:

1) для всякого $n>0$ системе $\Sigma$ принадлежит не более чем конечное число $n$-мерных параллелоэдров;

2) для всякого $n>0$ можно составить такое множество $\mathcal{P}_{1}, \mathcal{P}_{2}, \ldots, \mathcal{P}_{\xi}$ афффинных образов параллелоэдров различных размерностей из системы $\Sigma$, что любой $n$-мерный параллелоэдр $\mathcal{P}$ с точностью до аффинного преобразования представим в виде

$$
\mathcal{P}=\lambda_{1} \mathcal{P}_{1} \oplus \lambda_{2} \mathcal{P}_{2} \oplus \cdots \oplus \lambda_{\xi} \mathcal{P}_{\xi},
$$

где $\lambda_{1}, \lambda_{2}, \ldots, \lambda_{\xi}$ - неотрицательные вешественные числа, отвечаюшие параллелоэдру $\mathcal{P}$

3) никакой элемент системы $\Sigma$ (коренной параллелоэдр) не является суммой Минковского никаких других коренных параллелоэдров или их аффинных образов.

Основная теОРема. Множество систем коренных параллелоэдров не nycmo.

ДоКАЗАТЕЛЬСТво содержится в примере системы коренных параллелоэдров и базируется на следующей теореме. 
КРАЕУГОЛЬНАЯ ТЕОРЕМА. Пусть $n \Pi К \Phi ~ f=f(x)$ принадлежит замыканию $\operatorname{clos} \Delta$ произвольной области $\Delta$ арифметического L-типа $u$

$$
f=\sum_{i=1}^{w} \lambda_{i} \varphi_{k_{i}}, \quad \lambda_{i}>0, \quad i=\overline{1, w}
$$

где $\varphi_{k_{1}}, \varphi_{k_{2}}, \ldots, \varphi_{k_{w}}-$ некоторые реберные формы области $\Delta$. Пусть также $\mathcal{P}$ - параллелоэдр, соответствующий форме $f$, a $\mathcal{P}_{k_{i}}$, где $i=\overline{1, w},-$ параллелоэдры, соответствующие формам $\varphi_{k_{1}}, \varphi_{k_{2}}, \ldots, \varphi_{k_{w}}$. Тогда

$$
\mathcal{P}=\lambda_{1} \mathcal{P}_{k_{1}} \oplus \lambda_{2} \mathcal{P}_{k_{2}} \oplus \cdots \oplus \lambda_{w} \mathcal{P}_{k_{w}}
$$

\section{§2. Основные понятия (DV- и $L$-разбиения, область типа)}

2.1. DV-разбиение, DV-области. В работе [1] дано определение некоторого "многогранника аналитического пространства $n$ измерений", связанного с $n$ ПКФ.

ОПРЕДЕЛЕНИЕ 2.1. Многогранник $R$ (соответствуюший $n \Pi К \Phi f$ ) есть множество точек $\alpha$, заданных координатами $\left(\alpha_{1}, \alpha_{2}, \ldots, \alpha_{n}\right)$ в ортонормированном базисе $\mathcal{E}$ пространства $E^{n}$, удовлетворяющими неравенствам

$$
2 \alpha x^{T} \leqslant f(x), \quad x \in Z^{n}
$$

Вороной показал, что многогранник $R$ ограничен, имеет $n$ измерений, а также, что из бесконечной системы неравенств (2.1) независимых только конечное количество, т. е. многогранник $R$ определяется конечным набором неравенств

$$
2 \alpha l_{i}^{T} \leqslant l_{i} A l_{i}^{T}, \quad i=\overline{1, \tau}
$$

из системы неравенств (2.1).

Для удобства описания и изучения таких неравенств впоследствии [15] было введено понятие индекса. Индексом длины $n$ называется любой арифметический вектор длины $n$, состоящий из нулей и единиц.

Целочисленный вектор $v=\left(v_{1}, v_{2}, \ldots, v_{n}\right)$ называется сравнимымм с индексом $J=\left(j_{1}, j_{2}, \ldots, j_{n}\right)$, если $v_{m} \equiv j_{m}(\bmod 2), m=\overline{1, n}$.

Представление минимума ПКФ $f$ по индексу $J \neq 0$ есть вектор $v$ (сравнимый с индексом $J)$, на котором ПКФ $f$ принимает значение, минимальное среди значений данной ПКФ на векторах, сравнимых с индексом $J$.

$\Gamma . \Phi$. Вороной также показал $[1, \S 48]$, что вектор $l_{i}$ определяет независимое неравенство из набора $(2.2)$, т. е., в частности, является нормалью $(n-1)$-мерной грани многогранника $R$ тогда и только тогда, когда ПК $f$ по индексу $J \equiv l_{i}$ $(\bmod 2)$ не имеет представлений минимума, кроме векторов $\pm l_{i}$. Эти векторы $l_{i}$ из неравенств (2.2) назьваются $l$-векторами соответствующей ПКФ $f$ и многогранника $R$. Для грани многогранника $R$, которая определяется уравнением $2 \alpha l_{i_{0}}^{T}=l_{i_{0}} A l_{i_{0}}^{T}$, вектор $l_{i_{0}}$ мы называем определяющ, $и$ м $l$-вектором. Совокупность 
определяющих $l$-векторов граней, в пересечении дающих некоторую вершину $\alpha^{*}$ многогранника $R$, мы называем определяющими l-векторами этой вершины.

Отметим, что вместе с каждым неравенством $2 \alpha l_{i}^{T} \leqslant l_{i} A l_{i}^{T}$ в систему (2.2) входит и неравенство $2 \alpha\left(-l_{i}\right)^{T} \leqslant l_{i} A l_{i}^{T}$. Такие два неравенства можно объединить в одно: $2\left|\alpha l_{i}^{T}\right| \leqslant l_{i} A l_{i}^{T}$.

$\Gamma$.. Вороной доказывает, что, выполняя переносы многогранника $R$, определенного неравенствами (2.2), вдоль векторов $\lambda=t A$, где $t$ - произвольный целочисленный вектор (произвольная целочисленная строка), мы получим множество многогранников, образующих разбиение всего пространства, которое наиболее естественно называть разбиением Дирихле-Вороного (DV-разбиением, отвечающим $n \Pi K \Phi f)$. Таким образом, многогранник $R$, соответствующий ПК $\Phi f$, является параллелоэдром. Каждый элемент DV-разбиения (в том числе и многогранник $R$ ) мы будем далее называть DV-областью (соответствующей ПК $\Phi f)$.

Говорят, что параллелоэдр, полученный из DV-области $R$ переносом на вектор $\lambda=t A, t \in Z^{n}$, характеризуется вектором $t$. Параллелоэдр $R$ мы будем называть исходным.

В том случае, если DV-разбиение, отвечающее $n \Pi K \Phi f$, является примитивным, ПК $\Phi$ и соответствующий параллелоэдр также называют примитивны.ми.

2.2. $L$-разбиение. Для примитивных $n \Pi K \Phi$, кроме DV-разбиения, определяется еше одно разбиение ( $L$-разбиение).

ОПРЕДЕЛЕНИЕ 2.2 (Г.Ф. Вороной). Вершине примитивного DV-разбиения, в которой сходятся параллелоэдры, характеризуюшиеся векторами $t_{1}, t_{2}, \ldots, t_{n+1}$, ставится в соответствие симплекс с вершинами в точках $t_{1}, t_{2}, \ldots, t_{n+1}$. Такие симплексы называются $L$-симплексами.

Вороной доказал, что $L$-симплексы, соответствующие заданной $n \Pi K \Phi$, в совокупности образуют разбиение грань-в-грань пространства $E^{n}$. Далее это разбиение мы будем называть $L$-разбиением, отвечающим заданной ПКФ. Замечательно, что разность $t-t^{\prime}$, где $t$ и $t^{\prime}$ - векторы, характеризующие два параллелоэдра, смежных друг с другом по $(n-1)$-мерной грани, представляет собой $l$-вектор; другими словами, одномерный остов $L$-разбиения состоит из копий $l$-векторов. Это позволяет говорить об $l$-векторах многогранника $R$ как об $l$-векторах каждого из рассматриваемых параллелоэдров.

Доказывается, что DV - и $L$-разбиения, соответствуюшие одной и той же ПК комбинаторно-метрически дуальны [1], [14]. Из сказанного достаточно ясно [1], что двум примитивным формам $f$ и $f^{\prime}$, которым соответствует одно и то же $L$-разбиение, отвечают “одинаково устроенные" DV-разбиения. А именно, наборы $l$-векторов для этих двух форм совпадают, т. е. параллелоэдры $\mathcal{P}$ и $\mathcal{P}^{\prime}$, отвечающие данным формам, определяются неравенствами вида (2.2) с одними и теми же коэффищиентами. Кроме того, вершины параллелоэдров находятся в таком взаимно однозначном соответствии, что соответствующие вершины $\alpha \in \mathcal{P}$ и $\alpha^{\prime} \in \mathcal{P}^{\prime}$ имеют одни и те же определяющие $l$-векторы. Вершины этих параллелоэдров, находящиеся в указанном соответствии, мы будем называть когерентными (согласованньцми). 
2.3. Области типа. Областью $\Delta \subset \mathbb{K}^{N} \subset \mathbb{E}^{N}$ [1]-[5], [14], [15] арифметического $L$-типа примитивных параллелоэдров называется множество $n \Pi K \Phi$ с одним и тем же $L$-разбиением.

Еше раз отметим, что мы работаем в системе определений Вороного. О взаимосвязи этих определений и определений, данных Б. Н. Делоне и Б. А. Венковым, cM. [14].

$\Gamma$.. . Вороной показал, что каждая область $\Delta$ является открытым бесконечным выпуклым полиэдральным конечногранным конусом с вершиной в точке $\mathcal{O}$.

Если рассматривать пересечения вида $\cos \Delta \cap \mathbb{K}^{N}$, то все они как замкнутые относительно конуса $\mathbb{K}^{N}$ многогранники образуют классическое разбиение грань-в-грань конуса $\mathbb{K}^{N}$ (см. [1]).

Каждой области $\Delta$ соответствует набор реберных форм $\varphi_{1}, \varphi_{2}, \ldots, \varphi_{s}$ (из каждого ребра области $\Delta$ выбрана одна ПКФ). Вообще говоря, некоторые из этих форм положительно, а некоторые - неотрицательно определены. Таким образом, любая ПКФ $f \in \Delta$ выражается в виде

$$
f=\sum \sum a_{i j} x_{i} x_{j}=\sum_{k=1}^{s} u_{k} \varphi_{k}, \quad u_{k} \geqslant 0, \quad k=\overline{1, s} .
$$

В таком же виде выражается и любая форма $f \in \operatorname{clos} \Delta$. При этом форма, лежащая на границе области $\Delta$, может быть, вообще говоря, как положительно, так и неотрицательно определенной.

Каждая целочисленная унимодулярная замена переменных приводит к такому аффинному преобразованию пространства $\mathbb{E}^{N}$, при котором как конус положительности $\mathbb{K}^{N}$, так и его разбиение на области арифметического $L$-типа переходят в себя.

Областью геометрического L-типа называется совокупность попарно целочисленно унимодулярно эквивалентных областей арифметического $L$-типа [14]. Далее типом формы мы называем ее арифметический $L$-тип.

Доказательство следуюшей леммы можно найти в [16], но здесь мы приведем более простое доказательство, основанное на формуле, завершающей $§ 86$ работы [1].

Лемма 2.1. Пусть квадратичная форма $f \in \operatorname{int} \Delta^{\prime}$, где $\Delta^{\prime}$ - грань некоторой области типа $\Delta$, имеет ранг $k \leqslant n$. Тогда существует такое челочисленное унимодулярное преобразование переменных, после применения которого все формы, принадлежсащие образу $\Delta^{\prime}$, зависят (существенно) только от первых $k$ переменных $x_{1}, \ldots, x_{k}$.

ДокАЗАТЕльСтво. Пусть $\varphi_{i_{1}}, \varphi_{i_{2}}, \ldots, \varphi_{i_{\tau}}$ - реберные формы грани $\Delta^{\prime}$. Тогда согласно указанной формуле Вороного

$$
f=\sum_{j=1}^{\tau} \mu_{j} \varphi_{i_{j}}=\sum_{j=1}^{\tau} \mu_{j} \sum_{r=1}^{\sigma} \lambda_{r}^{i_{j}}\left(p_{1 r} x_{1}+p_{2 r} x_{2}+\cdots+p_{n r} x_{n}\right)^{2},
$$

где все числа $p_{i r}, i=\overline{1, n}, r=\overline{1, \sigma},-$ целые, а все коэффициенты $\lambda_{r}^{i_{j}}$ и $\mu_{j}$ можно всегда считать положительньми. Точно в таком же виде, но с другими коэффициентами $\mu_{j}>0, j=\overline{1, \tau}$, представима и любая другая форма из int $\Delta^{\prime}$. 
Произведя суммирование по $j$ в формуле (2.3), получаем

$$
f=\sum_{r=1}^{\sigma} \nu_{r}\left(p_{1 r} x_{1}+p_{2 r} x_{2}+\cdots+p_{n r} x_{n}\right)^{2}
$$

Обозначим через $P=\left(p_{i j}\right)$ матрицу (с $\sigma$ строками и $n$ столбцами) коэффициентов из этого представления. Во-первых, докажем, что ее ранг равен $k$. Положим ее ранг равным $k^{\prime}$ и докажем, что $k^{\prime}=k$. Будем считать, что первые $k^{\prime}$ строк матрицы $P$ линейно независимы. Произведем невырожденное линейное преобразование

$$
\begin{aligned}
& y_{1}=p_{11} x_{1}+\cdots+p_{n 1} x_{n}, \\
& \ldots \ldots \ldots \ldots \cdots \cdots \cdots \cdots \\
& y_{k^{\prime}}=p_{1 k^{\prime}} x_{1}+\cdots+p_{n k^{\prime}} x_{n}, \\
& y_{k^{\prime}+1}=p_{k^{\prime}+1}(x) \\
& \cdots \cdots \cdots \cdots \cdots \cdots \\
& y_{n}=p_{n}(x)
\end{aligned}
$$

где $p_{k^{\prime}+1}(x), \ldots, p_{n}(x)$ - такие произвольные линейные формы от $x_{1}, x_{2}, \ldots, x_{n}$, которые делают преобразование невырожденным. Поскольку ранг полученной из $P$ матрицы по-прежнему равен $k^{\prime}$ (в первых $k^{\prime}$ строках ненулевые элементы только единицы на главной диагонали), то последние $n-k^{\prime}$ столбцов всей матрицы заполнены нулями и сумма квадратов соответствующих этой матрище линейных форм имеет ранг $k^{\prime}$. Однако такая сумма в силу (2.4) имеет ранг $k$. Таким образом, $k^{\prime}=k$.

Известно (Г. Минковский), что целочисленную матрицу ранга $k$, первые $k$ строк которой линейно независимы, можно целочисленным унимодулярным преобразованием привести к следующему виду:

$$
P^{\prime}=\left\|\begin{array}{cccccc}
p_{11}^{\prime} & 0 & 0 & 0 & \ldots & 0 \\
\vdots & \ddots & 0 & 0 & \ldots & 0 \\
p_{k 1}^{\prime} & \ldots & p_{k k}^{\prime} & 0 & \ldots & 0 \\
p_{(k+1) 1}^{\prime} & \ldots & p_{(k+1) k}^{\prime} & 0 & \ldots & 0 \\
\ldots \ldots \ldots \ldots \ldots & \ldots \ldots & \ldots \ldots & \ldots \\
p_{\sigma 1}^{\prime} & \ldots & p_{\sigma k}^{\prime} & 0 & \ldots & 0
\end{array}\right\| .
$$

Преобразование, переводящее $P$ в $P^{\prime}$ и является искомым. Лемма доказана.

Отсюда, в частности, следует, что каждой открытой грани каждой области арифметического типа можно приписать два целых числа: размерность грани и ранг (неотрицательных) квадратичных форм, отвечающих точкам, из которых она состоит. 


\section{§ 3. Выход области типа на границу конуса положительности}

3.1. О гранях ранга $k \leqslant n$. Зафиксируем до конца $\S 5$ область $\Delta$ арифметического $L$-типа $n \Pi K \Phi$.

Пусть форма $f_{\omega}$ с матрицей $A_{\omega}$ лежит на грани $\Delta^{\prime} \subset \Delta$ и имеет ранг $k \leqslant n$. Пусть также $\operatorname{dim} \Delta^{\prime}<N$. Будем считать, что уже произведена такая замена переменных, что форма $f_{\omega}$ зависит лишь от первых $k$ переменных.

Наряду с формой $f_{\omega}$ мы будем рассматривать форму $\tilde{f}_{\omega}=\tilde{f}_{\omega}\left(x_{1}, x_{2}, \ldots, x_{k}\right)$ c $(k \times k)$-матрицей $\tilde{A}_{\omega}$, которая при $k<n$ получается из матрицы $A_{\omega}$ удалением последних (нулевых) $n-k$ строк и $n-k$ столбцов, а при $k=n$ совпадает с матрищей $A_{\omega}$. Как DV-разбиение, так и $L$-разбиение, соответствуюшие $k \Pi K \Phi \tilde{f}_{\omega}$, будем считать заданными в $E^{k}$. Исходный параллелоэдр такого DV-разбиения будем обозначать через $\mathcal{P}_{\omega}$.

Зафиксируем до конца $\S 4$ произвольную последовательность ПК $\Phi\left\{f_{q}\right\}_{q=1}^{\infty}=$ $\left\{f_{q}\right\} \subset \Delta$ (форме $f_{q}$ соответствует матрица $A_{q}$ и исходный параллелоэдр $\mathcal{P}_{q}$, где $q=1,2, \ldots)$, для которой

$$
\left\|A_{\omega}-A_{q}\right\| \leqslant \frac{1}{q}, \quad q=1,2, \ldots
$$

Введем обозначения: $g_{q}=f_{q}-f_{\omega}$ и $B_{q}=A_{q}-A_{\omega}$. В таком случае для произвольного вектора $x \in \mathbb{R}^{n}$ справедливы оценки

$$
\left|g_{q}(x)\right|=\left|x B_{q} x^{T}\right| \leqslant|x|\left|B_{q} x^{T}\right| \leqslant|x|\left\|B_{q}\right\||x| \leqslant \frac{|x|^{2}}{q} .
$$

Очевидно, имеем

$$
f_{\omega}=\lim _{q \rightarrow \infty} f_{q} \text { и } A_{\omega}=\lim _{q \rightarrow \infty} A_{q} .
$$

Одной из дальнейших промежуточных целей будет доказательство аналога этих равенств для параллелоэдров.

3.2. Леммы. Вместе с каждым вектором $v=\left(v_{1}, v_{2}, \ldots, v_{n}\right)$ будем рассматривать векторы $\tilde{v}, \hat{v}$ и $\check{v}$, определенные следуюшим образом:

$$
\begin{aligned}
& \tilde{v}=\left(v_{1}, v_{2}, \ldots, v_{k}\right) \in E^{k}, \\
& \hat{v}=\left(v_{1}, v_{2}, \ldots, v_{k}, 0,0, \ldots, 0\right) \in E^{n}, \\
& \check{v}=\left(0,0, \ldots, 0, v_{k+1}, \ldots, v_{n}\right) \in E^{n} .
\end{aligned}
$$

В случае $k=n$ полагаем $\tilde{v}=\hat{v}=v, \check{v}=\overline{0}$. Очевидно, $\hat{v}$ и $\check{v}$ суть проекции вектора $v$ на подпространство $E^{k}$ и его ортогональное дополнение соответственно, а вектор $\tilde{v}$ - проекция $v$ на $E^{k}$, рассматриваемая как вектор пространства $E^{k}$.

ЛЕмма 3.1 (о представлениях минимума граничной формы). Пусть $l=$ $\left(l_{1}, \ldots, l_{k}, \ldots, l_{n}\right)$ - представление минимума некоторой ПКФ $f \in \Delta$ по индексу $J=\left(j_{1}, \ldots, j_{k}, \ldots, j_{n}\right)$. Тогда $\tilde{l}=\left(l_{1}, \ldots, l_{k}\right)$ - представление минимума формыл $\tilde{f}_{\omega}$ по индексу $\tilde{J}=\left(j_{1}, \ldots, j_{k}\right)$. 
ДоКАЗАТЕЛЬСТвО. Поскольку представления минимума всех ПКФ из области $\Delta$ по одному и тому же индексу совпадают между собой, представлением минимума любой ПКФ $f_{q} \in\{f\}$ по индексу $J$ является вектор $\left(l_{1}, \ldots, l_{n}\right)$. Зафиксируем произвольньй вектор $t$, сравнимый с индексом $J$. Вектор $l=\left(l_{1}, \ldots, l_{n}\right)$ является представлением минимума формы $f_{q}$ по индексу $J$, следовательно, для вектора $t$ выполнено соотношение $f_{q}(l) \leqslant f_{q}(t)$, т. е. $f_{\omega}(l)+g_{q}(l) \leqslant f_{\omega}(t)+g_{q}(t)$ или $\tilde{f}_{\omega}(\tilde{l})+g_{q}(l) \leqslant \tilde{f}_{\omega}(\tilde{t})+g_{q}(t)$, и

$$
\tilde{f}_{\omega}(\tilde{l}) \leqslant \tilde{f}_{\omega}(\tilde{t})+\left(g_{q}(t)-g_{q}(l)\right) .
$$

Имеем $\left|g_{q}(t)\right| \leqslant \frac{|t|^{2}}{q}$ и $\left|g_{q}(l)\right| \leqslant \frac{|l|^{2}}{q}$, т.е. $g_{q}(t)-g_{q}(l) \leqslant \frac{1}{q}\left(|t|^{2}+|l|^{2}\right)$, и

$$
\tilde{f}_{\omega}(\tilde{l}) \leqslant \tilde{f}_{\omega}(\tilde{t})+\frac{1}{q}\left(|t|^{2}+|l|^{2}\right)
$$

Поскольку неравенство (3.1) выполнено для всех натуральных $q$, имеем

$$
\tilde{f}_{\omega}(\tilde{l}) \leqslant \tilde{f}_{\omega}(\tilde{t})
$$

В силу произвольности выбора вектора $t$ неравенство (3.2) справедливо для любого вектора $\tilde{t}$, сравнимого с индексом $\tilde{J}$. Лемма доказана.

ЛЕмма 3.2 (о представлениях минимума по “хвостовым" индексам). Пусть $k<n$. По индексу $J=\left(0, \ldots, 0, j_{k+1}, \ldots, j_{n}\right)$ для каждой ПКФ $f \in \Delta$ представлением минимума является вектор из ортогонального дополнения $\kappa$ подпространству $E^{k}$, m. е. вектор вида $\left(0, \ldots, 0, l_{k+1}, \ldots, l_{n}\right)$.

ДокАЗАТЕЛьство. Как и прежде, рассмотрим последовательность $\left\{f_{q}\right\}$. Пусть $l=\left(l_{1}, \ldots, l_{n}\right)$ - представление минимума форм $f_{q} \in\left\{f_{q}\right\}$ по индексу $J$.

Рассмотрим произвольный вектор $t$ вида $\left(0, \ldots, 0, t_{k+1}, \ldots, t_{n}\right)$, сравнимый с индексом $J$. Так же, как в лемме 3.1 , получаем

$$
\tilde{f}_{\omega}(\tilde{l}) \leqslant \tilde{f}_{\omega}(\tilde{t})+\frac{1}{q}\left(|t|^{2}+|l|^{2}\right)
$$

а с учетом того, что $\tilde{t}=\overline{0}$, имеем

$$
\tilde{f}_{\omega}(\tilde{l}) \leqslant \frac{1}{q}\left(|t|^{2}+|l|^{2}\right) .
$$

Поскольку число $\frac{1}{q}$ можно сделать сколь угодно мальм, получаем $f_{\omega}(\tilde{l}) \leqslant 0$. Учитывая, что $\tilde{f}_{\omega}-$ положительно определенная форма от $k$ переменных, получаем, что $\tilde{f}_{\omega}(\tilde{l})=0$ и $\tilde{l}=\overline{0}$. Лемма доказана. 
ЛЕмма 3.3. Совокупность векторов - представлений минимума произвольно выбранной $n \Pi К \Phi ~ f \in \Delta$ по всем индексам вида $J=\left(0, \ldots, 0, j_{k+1}, \ldots\right.$ $\left.\ldots, j_{n}\right)$ - имеет ранг $n-k$.

ДокАЗАТЕЛЬСТвО. Пусть $k=n$. Тогда утверждение леммы тривиально, поскольку рассматриваемое множество индексов пусто.

Рассмотрим случай $k<n$. Согласно лемме 3.2 представления минимума $\Pi K \Phi f \in \Delta$ по всем индексам указанного вида суть представления минимума $(n-k) \Pi K \Phi f_{*}\left(x_{k+1}, \ldots, x_{n}\right)=f\left(0, \ldots, 0, x_{k+1}, \ldots, x_{n}\right)$ по всем возможным индексам, и утверждение леммы следует из того, что DV-области, соответствующие $(n-k) \Pi K \Phi$, имеют размерность $n-k$. Лемма доказана.

Далее понадобятся две следующие известные леммы из линейной алгебры, которые мы приведем в нужной нам формулировке.

Лемма 3.4. Пусть $\left\{u_{1}, u_{2}, \ldots, u_{\mu}\right\}$ - система векторов в пространстве $E^{m}$, имеющая ранг $m, u v \in E^{m}$ - некоторый вектор. Пусть также $\left|v u_{i}^{T}\right|<\varepsilon, i=\overline{1, \mu} ;$ тогда $|v|<\varepsilon C$, где $C$ - константа, зависящая только om сuстемы $\left\{u_{i}\right\}$.

Очевидно.

ЛЕмма 3.5. Пусть $\left\{u_{1}, u_{2}, \ldots, u_{\mu}\right\} \subset E^{n}$ - система векторов ранга $n$, $P \subset E^{n}$ - подпространство размерности $k \leqslant n u d_{i}$ - проекиия вектора $u_{i}$, где $i=\overline{1, \mu}$, на Р. Тогда система векторов $\left\{d_{1}, d_{2}, \ldots, d_{\mu}\right\}$ имеет ранг $k$.

Очевидно.

ЛЕмма 3.6 (о "трубочке"). Пусть ПК $\Phi f_{q} \in\left\{f_{q}\right\}$, и пусть $\mathcal{P}_{q}$ - параллелоәдр, соответствующий әтой ПКФ, т. е. определенный (ср. с (2.2)) системой неравенств

$$
2 \alpha l_{i}^{T} \leqslant l_{i} A_{q} l_{i}^{T}, \quad i=\overline{1, \tau} .
$$

Пусть также $\alpha^{*}=\left(\alpha_{1}, \alpha_{2}, \ldots, \alpha_{n}\right) \in \mathcal{P}_{q}$. Тогда

$$
\left|\tilde{\alpha}^{*}\right| \leqslant \frac{C_{\Delta}}{q},
$$

где $C_{\Delta}-$ константа, зависящая только от выбора области $\Delta$.

ДокАЗАТЕЛЬСтво. В случае $k=n$ очевидно, что $\alpha^{*}=\overline{0}$, и утверждение леммы тривиально. Далее считаем $k<n$.

Пусть $l_{i_{1}}, \ldots, l_{i_{\mu}}$ - представления минимума формы $f_{q}$ по индексам вида $(0, \ldots$ $\left.\ldots, 0, j_{k+1}, \ldots, j_{n}\right)$. Согласно лемме 3.2 каждый такой вектор $l_{i_{h}}$ имеет вид $(0, \ldots$ $\left.\ldots, 0, l_{i_{h} k+1}, \ldots, l_{i_{h} n}\right)$.

Поскольку точка $\alpha^{*}$ принадлежит параллелоэдру $\mathcal{P}_{q}$, она удовлетворяет неравенствам (3.3), в частности $\left|2 \alpha^{*} l_{i_{h}}^{T}\right| \leqslant l_{i_{h}} A_{q} l_{i_{h}}^{T}$ при $h=\overline{1, \mu}$, т. е. $\left|2\left(\hat{\alpha^{*}}+\tilde{\alpha}^{*}\right) l_{i_{h}}^{T}\right| \leqslant$ $l_{i_{h}} A_{q} l_{i_{h}}^{T}$, а следовательно, и $\left|2 \tilde{\alpha}^{*} l_{i_{h}}^{T}\right| \leqslant l_{i_{h}} B_{q} l_{i_{h}}^{T}$, поскольку $\hat{\alpha}^{*} l_{i_{h}}^{T}=0$ и $l_{i_{h}} A_{\omega} l_{i_{h}}^{T}=0$ при $h=\overline{1, \mu}$. Так как

$$
l_{i_{h}} B_{q} l_{i_{h}}^{T} \leqslant \frac{\left|l_{i_{h}}\right|^{2}}{q} \leqslant \frac{\max _{h}\left|l_{i_{h}}\right|^{2}}{q}
$$


а система векторов $l_{i_{h}}$ имеет ранг $n-k$, то, учитывая лемму 3.4 , получаем, что

$$
\left|\tilde{\alpha^{*}}\right|<\frac{\max _{h}\left|l_{i_{h}}\right|^{2} C_{\Delta^{\prime}}}{q}
$$

Здесь константа $C_{\Delta^{\prime}}$ зависит только от выбора той грани $\Delta^{\prime}$ области $\Delta$, которой принадлежит форма $f_{\omega}$. Поскольку область $\Delta$ имеет конечное число граней, получаем $\left|\check{\alpha^{*}}\right|<\frac{C_{\Delta}}{q}$, где $C_{\Delta}=\max _{\Delta^{\prime} \subset \operatorname{clos} \Delta} C_{\Delta^{\prime}} \max \left|l_{i}\right|^{2}$. Лемма доказана.

Таким образом, мы показали, что для любого цилиндра ( "трубочки") с "осью" пространством $E^{k}$ и "направляющей” - сфферой $S^{n-k-1}$, имеющей центр в начале координат и расположенной в ортогональном дополнении $E^{n-k}$ к пространству $E^{k}$, начиная с некоторого номера $q$ все параллелоэдры $\mathcal{P}_{r}, r \geqslant q$, лежат внутри этого цилиндра.

3.3. $L$-конусы вершин. Рассмотрим два важных определения.

ОПРЕДЕЛЕНИЕ 3.1. Вершинным конусом вершины $\alpha^{0}$ параллелоэдра $\mathcal{P}$ мы назовем множество точек $\{\beta\}$, удовлетворяющих неравенствам

$$
2\left(\beta-\alpha^{0}\right) l_{j_{k}}^{T} \leqslant 0
$$

где $k=\overline{1, m}$ и $l_{j_{1}}, l_{j_{2}}, \ldots, l_{j_{m}}$ - определяюшие векторы вершины $\alpha^{0}$.

Отметим, что неравенства (3.4) заменой $\alpha=\beta-\alpha^{0}$ приводятся к виду

$$
2 \alpha l_{j_{k}}^{T} \leqslant 0, \quad k=\overline{1, m}
$$

и что вершиной конуса, определяемого неравенствами (3.4), является точка $\alpha^{0}$, а вершиной конуса, определяемого неравенствами (3.5), - начало координат. В дальнейшем, говоря о вершинном конусе, мы будем подразумевать конус с вершиной в начале координат.

ОПРЕДЕлЕНИЕ $3.2 . \quad L$-конусом вершины $\alpha^{0}$ параллелоэдра $\mathcal{P}$ мы назовем множество точек $\{x\}$, определяемых равенствами

$$
x=\sum_{k=1}^{m} \rho_{k} l_{j_{k}}, \quad \rho_{k} \geqslant 0, \quad k=\overline{1, m}
$$

где $l_{j_{1}}, l_{j_{2}}, \ldots, l_{j_{m}}$ - определяюшие векторы вершины $\alpha^{0}$.

ПримеР 3.1. Для примитивного параллелоэдра $L$-конус вершины - это многогранный угол соответствуюшего ей $L$-симплекса с вершиной в начале координат.

Заметим, что оба наших конуса определены для вершин не только примитивного параллелоэдра.

С учетом приведенного примера из того, что $L$-симплексы образуют разбиение пространства, следует, например, теорема. 
Tеорема 3.1. Совокупность L-конусов вершин примитивного параллелоэдра образует разбиение пространства.

Из дальнейшего (см. доказательство леммы 3.11) будет ясно, что этот факт имеет место и для непримитивных параллелоэдров.

ЛЕмма 3.7 (о нормалях из $L$-конуса). Пусть $\mathcal{L}$ - это $L$-конус вериины $\alpha^{0}$, определенный соотношениями (3.6). Пусть также вектор $h$ лежит внутри конуса $\mathcal{L}$, а вектор $v$-вне этого конуса. Тогда неравенство $\alpha h^{T} \leqslant 0$ является следствием неравенств (3.5), а неравенство $\alpha v^{T} \leqslant 0$ - не является.

Иными словами, плоскость, проходящая через начало координат и ортогональная вектору $v$, пересекает вершинный конус по внутренним точкам, а плоскость, ортогональная вектору $h$, не пересекает.

Доказательство следует, например, из [2, § 14].

Неравенство (2.2), соответствуюшее нормали из внутренности $L$-конуса, будем называть существенно зависимым от неравенств (3.4). Граничная плоскость полупространства, определяемого этим неравенством, является опорной для вершинного конуса и имеет с ним единственную обшую точку - вершину конуса.

СлЕДСТВИЕ 3.1. Два L-конуса, соответствующие различным вериинам одного и того же параллелоэдра (не обязательно примитивного), не имеют общих внутренних точек.

Здесь достаточно заметить, что внутренней точке $L$-конуса соответствует плоскость, проходящая через соответствующую вершину и не касающаяся более никакой грани параллелоэдра.

Другим несложным следствием леммы 3.7 и результатом применения к параллелоэдрам достаточно известного обшего факта является следующая

Лемма 3.8. Пусть $\alpha$ - некоторая вершина параллелоэдра $\mathcal{P}$, а $\mathcal{K}$ - выпукльй многогранный конус с вершиной в начале координат. Пусть также $\mathcal{K}$ содержит в себе вершинный конус вершины $\alpha$. Тогда конус с вершиной в начале координат, натянутый на нормали к граням $\mathcal{K}$, содержится в $L$-конусе вериинь $\alpha$.

3.4. О вершинах и гранях параллелоэдров. Всюду далее при рассмотрении последовательностей $\left\{\alpha_{q}\right\}$ когерентных вершин подразумевается, что из каждого параллелоэдра $\mathcal{P}_{q} \in\left\{\mathcal{P}_{q}\right\}$ в последовательность входит одна и только одна вершина $\alpha_{q}$, и вершины в последовательности $\left\{\alpha_{q}\right\}$ пронумерованы в том же порядке, что и параллелоэдры в последовательности $\left\{\mathcal{P}_{q}\right\}$.

ЛЕмма 3.9 (первая лемма о происхождении вершин параллелоэдра $\mathcal{P}_{\omega}$ ). Пусть $l$-векторы $l_{i_{1}}, l_{i_{2}}, \ldots, l_{i_{n}}$ определяют последовательность $\left\{\alpha_{q}\left(l_{i_{1}}, l_{i_{2}}, \ldots\right.\right.$ $\left.\left.\ldots, l_{i_{n}}\right)\right\}$ когерентных вершин параллелоэдров $\mathcal{P}_{q}$. Тогда:

1) последовательность $\left\{\alpha_{q}\left(l_{i_{1}}, l_{i_{2}}, \ldots, l_{i_{n}}\right)\right\}$ при $q \rightarrow \infty$ сходится $\kappa$ некоторой точке $\alpha_{\omega} \in E^{k} \subset E^{n}$;

2) точка $\alpha_{\omega}$ является верииной параллелоэдра $\mathcal{P}_{\omega}$; 
3) для точки $\alpha_{\omega}$ справедливы равенства

$$
\begin{gathered}
2 \tilde{\alpha}_{\omega} \tilde{l}_{i_{j}}^{T}=\tilde{l}_{i_{j}} \tilde{A}_{\omega} \tilde{l}_{i_{j}}^{T}, \quad j=\overline{1, n}, \\
\check{\alpha}_{\omega}=\overline{0} ;
\end{gathered}
$$

4) точка $\alpha_{\omega}$ әтими равенствами однозначно определяется.

ДокАЗАТЕЛЬСтво. Представим каждую вершину $\alpha_{q} \in\left\{\alpha_{q}\left(l_{i_{1}}, l_{i_{2}}, \ldots, l_{i_{n}}\right)\right\}$ в виде суммы $\hat{\alpha}_{q}+\check{\alpha}_{q}$.

Для каждой такой вершины $\alpha_{q}$ справедливы равенства

$$
2 \alpha_{q} l_{i_{j}}^{T}=l_{i_{j}} A_{q} l_{i_{j}}^{T}, \quad j=\overline{1, n} .
$$

Из (3.7) с учетом того, что $A_{q}=A_{\omega}+B_{q}$, получаем

$$
\begin{array}{ll}
2\left(\hat{\alpha}_{q}+\check{\alpha}_{q}\right) l_{i_{j}}^{T}=l_{i_{j}} A_{\omega} l_{i_{j}}^{T}+l_{i_{j}} B_{q} l_{i_{j}}^{T}, & j=\overline{1, n}, \\
2 \hat{\alpha}_{q} l_{i_{j}}=l_{i_{j}} A_{\omega} l_{i_{j}}^{T}+l_{i_{j}} B_{q} l_{i_{j}}^{T}-2 \check{\alpha}_{q} l_{i_{j}}^{T}, & j=\overline{1, n},
\end{array}
$$

и, далее,

$$
2 \tilde{\alpha}_{q} \tilde{l}_{i_{j}}^{T}=l_{i_{j}} A_{\omega} l_{i_{j}}^{T}+l_{i_{j}} B_{q} l_{i_{j}}^{T}-2 \check{\alpha}_{q} l_{i_{j}}^{T}, \quad j=\overline{1, n} .
$$

Для фиксированного $1 \leqslant j \leqslant n$ имеем $\lim _{q \rightarrow \infty} \check{\alpha}_{q}=\overline{0}$ и $\lim _{q \rightarrow \infty} \check{\alpha}_{q} l_{i_{j}}^{T}=0$ (из леммы 3.6), $\lim _{q \rightarrow \infty} l_{i_{j}} B_{q} l_{i_{j}}^{T}=0$ (так как $\left\|B_{q}\right\| \leqslant \frac{1}{q}$ ), значение $l_{i_{j}} A_{\omega} l_{i_{j}}^{T}$ постоянно. Следовательно, для каждого $1 \leqslant j \leqslant n$ числовая последовательность $\left\{\tilde{\alpha}_{q} \tilde{l}_{i_{j}}^{T}\right\}$ сходится к некоторому пределу. С учетом того, что множество векторов $\left\{\tilde{l}_{i_{j}}\right\}$ имеет ранг $k$, получаем, что и последовательность векторов $\left\{\tilde{\alpha}_{q}\right\}$ сходится, а значит, сходится и последовательность $\left\{\hat{\alpha}_{q}\right\}$ :

$$
\lim _{q \rightarrow \infty} \hat{\alpha}_{q}=\alpha_{\omega} \in E^{k}
$$

Для последовательности $\left\{\alpha_{q}\right\}$ получаем

$$
\lim _{q \rightarrow \infty} \alpha_{q}=\lim _{q \rightarrow \infty}\left(\hat{\alpha}_{q}+\check{\alpha}_{q}\right)=\alpha_{\omega}
$$

При этом $\check{\alpha}_{\omega}=\overline{0}$, т. е. точка $\alpha_{\omega}$ лежит в плоскости $E^{k}$.

Покажем, что точка $\alpha_{\omega}$ (или, что то же самое, $\left.\tilde{\alpha}_{\omega}\right)$ является вершиной параллелоэдра $\mathcal{P}_{\omega}$. Во-первых, $\alpha_{\omega}$ принадлежит параллелоэдру $\mathcal{P}_{\omega}$, так как для каждой точки $\alpha_{q}$ из последовательности, сходящейся к $\alpha_{\omega}$, выполнены все неравенства

$$
2 \alpha_{q} x^{T} \leqslant x A_{q} x^{T}, \quad x \in Z^{n},
$$

т.

$$
2 \alpha_{q} x^{T} \leqslant \tilde{x} \tilde{A}_{\omega} \tilde{x}^{T}+x B_{q} x^{T}, \quad x \in Z^{n}
$$


откуда предельным переходом получаем

$$
2 \tilde{\alpha}_{\omega} \tilde{x}^{T} \leqslant \tilde{x} \tilde{A}_{\omega} \tilde{x}^{T}, \quad \tilde{x} \in Z^{k}
$$

Аналогично, для всех $j=\overline{1, n}$ имеем

$$
2 \tilde{\alpha}_{\omega} \tilde{l}_{i_{j}}^{T}=\tilde{l}_{i_{j}} \tilde{A}_{i_{j}}^{T}
$$

Здесь следует отметить, что система векторов $\left\{\tilde{l}_{i_{j}}\right\}$ имеет ранг $k$ (из леммы 3.5 ). Таким образом, точка $\alpha_{\omega}$ удовлетворяет всем неравенствам (3.8), и при этом по крайней мере $k$ линейно независимых из них обращает в равенства (3.9). Следовательно, $\alpha_{\omega}$ - вершина параллелоэдра $\mathcal{P}_{\omega}$. Лемма доказана.

ЛЕмма 3.10. Пусть $\mathcal{P}_{q}$ - параллелоэдр из рассматриваемой последовательности и $\alpha_{q}$ - произвольная его вершина. Тогда проекция L-конуса $\mathcal{L}^{n}$ вериины $\alpha_{q}$ на плоскость $E^{k}$ лежит в L-конусе $\mathcal{L}^{k}$ некоторой веричны параллелоэдра $\mathcal{P}_{\omega} .\left(\right.$ (В случае $k=n$ такой проекцией $L$-конуса $\mathcal{L}^{n}$ является он сам.)

ДокАЗАТЕЛЬство. Пусть последовательность когерентных вершин, которой принадлежит вершина $\alpha_{q}$, определяется $l$-векторами $l_{i_{1}}, l_{i_{2}}, \ldots, l_{i_{n}}$. В соответствии с леммой 3.9 эта последовательность сходится к некоторой вершине $\alpha_{\omega}$ параллелоэдра $\mathcal{P}_{\omega}$, для которой справедливы неравенства (3.8) и равенства (3.9). Следовательно, для вершины $\alpha_{\omega}$ справедливы, в частности, неравенства

$$
2 \tilde{\alpha}_{\omega} \tilde{l}_{i_{j}}^{T} \leqslant \tilde{l}_{i_{j}} \tilde{A}_{\omega} \tilde{l}_{i_{j}}^{T}, \quad j=\overline{1, n}
$$

Каждое неравенство из системы (3.10) либо принадлежит к числу независимых неравенств, определяющих параллелоэдр $\mathcal{P}_{\omega}$, либо является следствием этих неравенств. Следовательно, выпуклый конус, определенный неравенствами

$$
2 \tilde{\alpha}_{\omega} \tilde{l}_{i_{j}}^{T} \leqslant 0, \quad j=\overline{1, n}
$$

содержит в себе вершинный конус вершины $\alpha_{\omega}$, и согласно лемме 3.8 это означает, что конус, определенный соотношениями

$$
x=\sum_{k=1}^{n} \rho_{k} \tilde{l}_{j_{k}}, \quad \rho_{k} \geqslant 0, \quad k=\overline{1, n}
$$

содержится в $L$-конусе вершины $\alpha_{\omega}$. Однако конус, определенный соотношениями (3.12), и есть проекция $L$-конуса вершины $\alpha_{q}$. Лемма доказана.

ОПРЕДЕЛЕНИЕ 3.3 . Последовательность $\left\{\alpha_{q}\left(l_{i_{1}}, l_{i_{2}}, \ldots, l_{i_{n}}\right)\right\}$ когерентных вершин, сходящуюся к вершине $\alpha_{\omega}$ параллелоэдра $\mathcal{P}_{\omega}$, мы называем порожсдающей для вершины $\alpha_{\omega}$. 
ЛЕмма 3.11 (вторая лемма о происхождении вершин параллелоэдра $\mathcal{P}_{\omega}$ ). Пусть $\alpha_{\omega}$ - произвольная вершина параллелоэдра $\mathcal{P}_{\omega}$. Тогда существует по крайней мере одна последовательность $\left\{\alpha_{q}\left(l_{i_{1}}, l_{i_{2}}, \ldots, l_{i_{n}}\right)\right\}$ когерентных вершин параллелоэдров $\mathcal{P}_{q} \in\left\{\mathcal{P}_{q}\right\}$, порождающая для вершины $\alpha_{\omega}$.

ДоКАЗАТЕЛЬСТво. Здесь под словом “проекция" всюду подразумевается ортогональная проекция на подпространство (плоскость) $E^{k}$.

Как уже отмечалось, $L$-конусы каждого параллелоэдра $\mathcal{P}_{q} \in\left\{\mathcal{P}_{q}\right\}$ образуют (одно и то же) разбиение пространства $E^{n}$. Значит, проекции этих $L$-конусов на $E^{k}$ заполняют это подпространство, и $L$-конус вершины $\alpha_{\omega}$ пересекается по внутренним точкам по крайней мере с одной такой проекцией. Обозначим $\left\{\beta_{q}\right\}$ соответствуюшую последовательность когерентных вершин параллелоэдров $\mathcal{P}_{q} \in\left\{\mathcal{P}_{q}\right\}$, а $\mathcal{L}_{\beta_{q}}$ - ее $L$-конус.

Согласно леммам 3.9 и 3.10 последовательность $\left\{\beta_{q}\right\}$ сходится к какой-то вершине $\beta_{\omega}$ параллелоэдра $\mathcal{P}_{\omega}$ и проекция $L$-конуса $\mathcal{L}_{\beta_{q}}$ лежит в $L$-конусе вершины $\beta_{\omega}$.

В соответствии со следствием $3.1 L$-конусы любых двух различных вершин параллелоэдра $\mathcal{P}_{\omega}$ не имеют общих внутренних точек. Поскольку проекция $L$-конуса $\mathcal{L}_{\beta_{q}}$ целиком лежит в $L$-конусе вершины $\beta_{\omega}$, эта проекция не может иметь обших внутренних точек с $L$-конусом никакой другой вершины параллелоэдра $\mathcal{P}_{\omega}$.

Таким образом, вершины $\beta_{\omega}$ и $\alpha_{\omega}$ совпадают, а последовательность $\left\{\beta_{q}\right\}$ является искомой последовательностью, сходящейся к вершине $\alpha_{\omega}$. Лемма доказана.

Обратим внимание, что небольшим изменением этого доказательства устанавливается справедливость для непримитивных параллелоэдров утверждения, аналогичного теореме 3.1 .

\section{§4. Параллелоэдр $\mathcal{P}_{\omega}$}

4.1. Сходимость последовательности множеств. Для удобства изложения мы приводим определение сходимости последовательности множеств в нужной нам форме.

ОПРЕДЕЛЕНИЕ 4.1. Последовательность $\left\{M_{i}\right\}$ множеств $M_{i} \subset E^{n}, i=1,2, \ldots$,

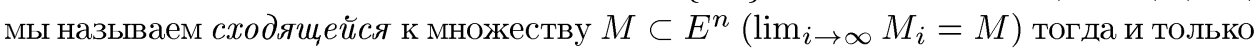
тогда, когда выполнены условия:

1) каждая точка $m \in M$ является пределом последовательности точек, взятых по одной из каждого множества последовательности $\left\{M_{i}\right\}$ :

$$
m \in M \Rightarrow \exists\left\{m_{i}\right\}_{i=1}^{\infty}: \quad m_{i} \in M_{i}, \quad i=1,2, \ldots, \quad m=\lim _{i \rightarrow \infty} m_{i}
$$

2) каждая сходящаяся последовательность точек, взятых не более чем по одной из каждого множества последовательности $\left\{M_{i}\right\}$, сходится к точке множества $M$ :

$$
\begin{gathered}
m_{k_{j}} \in M_{k_{j}}, \quad k_{1}<k_{2}<\ldots, \\
\lim _{j \rightarrow \infty} m_{k_{j}}=m \Rightarrow m \in M .
\end{gathered}
$$


4.2. Сходимость последовательности параллелоэдров. Докажем следуюшую теорему.

ТЕорема 4.1 (о предельном происхождении параллелоэдра $\mathcal{P}_{\omega}$ ). Имеет место соотношение

$$
\lim _{q \rightarrow \infty} \mathcal{P}_{q}=\mathcal{P}_{\omega}
$$

ДокаЗАТЕльство. Обозначим через $\mathcal{V}_{z}$ множество вершин параллелоэдра $\mathcal{P}_{z}$. Сначала докажем следующее:

$$
\lim _{q \rightarrow \infty} \mathcal{V}_{q}=\mathcal{V}_{\omega}
$$

1. Пусть $\alpha_{\omega} \in \mathcal{V}_{\omega}$, т. е. $\alpha_{\omega}$ - вершина параллелоэдра $\mathcal{P}_{\omega}$. В соответствии с леммой 3.11 существует последовательность когерентных вершин (т.е. точек, взятых по одной из каждого множества последовательности $\left\{\mathcal{V}_{q}\right\}$ ), сходящаяся к $\alpha_{\omega}$.

2. Рассмотрим произвольную сходящуюся последовательность $\left\{\beta_{q_{i}}\right\}$ точек, взятых не более чем по одной из каждого множества последовательности $\left\{\mathcal{V}_{q}\right\}$ (т.е. $\left.\beta_{q_{i}} \in \mathcal{V}_{q_{i}}, q_{1}<q_{2}<\ldots\right)$. Все точки всех множеств последовательности $\left\{\mathcal{V}_{q}\right\}$ распределены по последовательностям когерентных вершин, а этих последовательностей лишь конечное число. Поэтому из последовательности $\left\{\beta_{q_{i}}\right\}$ можно выбрать подпоследовательность, состояшую из точек одной и той же последовательности когерентных вершин.

Найденная подпоследовательность сходится к некоторой точке $\alpha_{\omega} \in \mathcal{V}_{\omega}$, следовательно, к этой же точке $\alpha_{\omega}$ сходится и последовательность $\left\{\beta_{q_{i}}\right\}$.

Итак, любая сходяшаяся последовательность точек из множеств последовательности $\left\{\mathcal{V}_{q}\right\}$ сходится к некоторой точке $\alpha_{\omega} \in \mathcal{V}_{\omega}$. Таким образом, доказано, что

$$
\lim _{q \rightarrow \infty} \mathcal{V}_{q}=\mathcal{V}_{\omega}
$$

3. Пусть $\mathcal{V}_{\omega}=\left\{\alpha_{\omega 1}, \ldots, \alpha_{\omega s}\right\}$. Рассмотрим произвольную точку $x_{\omega} \in \mathcal{P}_{\omega}$. Параллелоэдр является выпуклой оболочкой своих вершин, поэтому сушествуют такие неотрицательные числа $\mu_{1}, \mu_{2}, \ldots, \mu_{s}$, что $x_{\omega}=\sum_{i=1}^{s} \mu_{i} \alpha_{\omega i}$. Для каждой вершины параллелоэдра $\mathcal{P}_{\omega}$ зафикисируем по одной порождающей последовательности вершин параллелоэдров из последовательности $\left\{\mathcal{P}_{q}\right\}$. Пусть это последовательности $\left\{\alpha_{q 1}\right\},\left\{\alpha_{q 2}\right\}, \ldots,\left\{\alpha_{q s}\right\}$. Каждая точка $x_{q}=\sum_{i=1}^{s} \mu_{i} \alpha_{q i}$ принадлежит соответствующему параллелоэдру $\mathcal{P}_{q}$, и последовательность $\left\{x_{q}\right\}$ сходится к точке $x_{\omega}$.

4. Теперь рассмотрим произвольную сходящуюся последовательность $\left\{\gamma_{q_{i}}\right\}$ точек, взятых не более чем по одной из каждого параллелоэдра, принадлежащего последовательности $\left\{\mathcal{P}_{q}\right\}$. Предположим, она сходится к точке, не принадлежашей параллелоэдру $\mathcal{P}_{\omega}$. Тогда существует такая окрестность $O_{*}\left(\mathcal{P}_{\omega}\right) \subset E^{n}$, что начиная с некоторого номера $i_{*}$ точки последовательности $\left\{\gamma_{q_{i}}\right\}$ лежат вне этой окрестности.

С другой стороны, $\lim _{q \rightarrow \infty} \mathcal{V}_{q}=\mathcal{V}_{\omega}$. Следовательно, для любой сколь угодно малой окрестности $O\left(\mathcal{P}_{\omega}\right)$ параллелоэдра $\mathcal{P}_{\omega}$ сушествует номер $q^{\prime}$, начиная 
с которого все вершины параллелоэдра $\mathcal{P}_{q}$ лежат в окрестности $O\left(\mathcal{P}_{\omega}\right)$, а потому и $\mathcal{P}_{q} \subset O\left(\mathcal{P}_{\omega}\right)$, что при $O\left(\mathcal{P}_{\omega}\right) \subset O_{*}\left(\mathcal{P}_{\omega}\right)$ и достаточно больших $q_{i}$ приводит к противоречию, доказывающему ложность сделанного предположения.

Итак, любая сходящаяся последовательность точек из параллелоэдров последовательности $\left\{\mathcal{P}_{q}\right\}$ сходится к точке, принадлежашей параллелоэдру $\mathcal{P}_{\omega}$.

5. Таким образом, показано, что для последовательности $\left\{\mathcal{P}_{q}\right\}$ и параллелоэдра $\mathcal{P}_{\omega}$ выполнены оба условия из определения 4.1. Теорема доказана.

4.3. Расположение параллелоэдров ранга $k<n$. До сих пор мы не упоминали о различном расположении в пространстве $E^{n}$ параллелоэдров, соответствуюших граничным формам различного ранга.

Пусть квадратичная форма $f \in \operatorname{int} \Delta^{\prime}$, где $\Delta^{\prime}-$ грань некоторой области типа $\Delta$, имеет ранг $k \leqslant n$. В соответствии с леммой 2.1 существует такое целочисленное унимодулярное преобразование $P$ переменных формы (и отвечающее ему аффинное преобразование $\left.P: E^{n} \rightarrow E^{n}\right)$, что форма $f_{*}=P f$ зависит только от первых $k$ переменных. Так же, как и в п. 3.1, форма $\tilde{f}_{*}$ является положительно определенной формой от $k$ переменных, и поэтому ей соответствует параллелоэдр $\mathcal{P}_{*}^{k} \subset E^{k}$. Этот же параллелоэдр $\mathcal{P}_{*}^{k}$, но уже как множество точек в пространстве $E^{n}$, естественно называть параллелоэдром, соответствующим форме $f_{*}$. Аффинно эквивалентное ему множество $\mathcal{P}_{f}^{k}=P^{-1} \mathcal{P}$ является параллелоэдром в некотором $k$-мерном подпространстве пространства $E^{n}$.

ОПРЕДЕЛЕНИЕ 4.2. Построенное $k$-мерное множество $\mathcal{P}_{f}^{k} \subset E^{n}$ мы называем параллелоәдром, соответствующим квадратичной форме $f$.

Отметим, что все рассуждения из $\S 3,4$ инвариантны относительно целочисленных унимодулярных преобразований переменных. Следовательно, и теорема 4.1 справедлива для любой формы ранга $k \leqslant n$ (не обязательно приведенной к какому-то специальному виду), принадлежащей гранище какой-либо области арифметического $L$-типа.

Также отметим, что формам ранга $k$, лежашим на разных гранях одной и той же области $\Delta$ или на гранях различных областей, соответствуют параллелоэдры, лежащие, вообще говоря, в различных $k$-мерных подпространствах пространства $E^{n}$.

На рис. 1 с некоторой долей условности изображена последовательность двумерных параллелоэдров (шестиугольники), сходящаяся к одномерному параллелоэдру (отрезок). Соответствующая последовательность ПКФ выглядит так:

$$
\left\{f_{q}=\frac{1}{2 q}\left(x^{2}+y^{2}\right)+\frac{1}{2}(x+y)^{2}\right\}
$$

(см. также рис. 3)

\section{§5. О суммировании параллелоэдров по Минковскому}

ЛЕмма 5.1 (о пределе сумм Минковского). Пусть $\left\{M_{i}\right\}_{i=1}^{\infty} u\left\{N_{i}\right\}_{i=1}^{\infty}$ - ограниченные в совокупности последовательности компактных множеств, сходящиеся соответственно к множествам $M и N$, m.е.

$$
\lim _{i \rightarrow \infty} M_{i}=M, \quad \lim _{i \rightarrow \infty} N_{i}=N .
$$




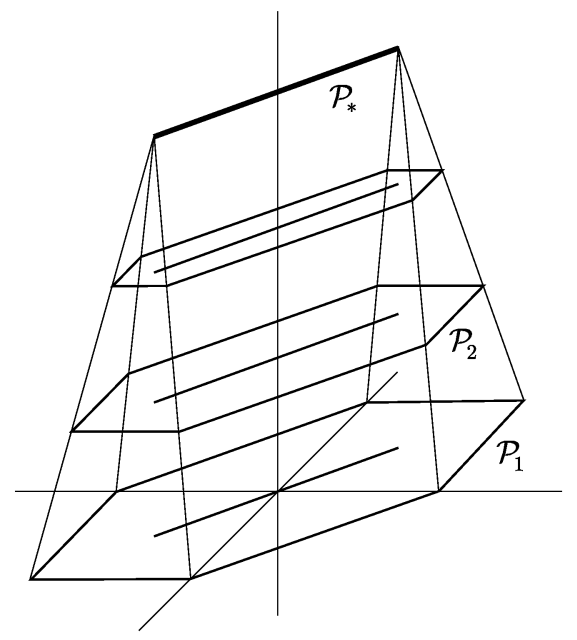

Рис. 1

Тогда $\left\{M_{i} \oplus N_{i}\right\}_{i=1}^{\infty}-$ последовательность множеств, сходящаяся $к$ множесmву $M \oplus N$, m.e.

$$
\lim _{i \rightarrow \infty} M_{i} \oplus N_{i}=M \oplus N
$$

ДоКАЗАТЕЛЬСТвО. Проверим для последовательности $\left\{M_{i} \oplus N_{i}\right\}_{i=1}^{\infty}$ оба условия из определения 4.1.

1. Пусть точка $\sigma$ принадлежит множеству $M \oplus N$ и $\sigma=\mu+\nu, \mu \in M, \nu \in N$. Существуют последовательности точек $\left\{\mu_{i}\right\}_{i=1}^{\infty}$ и $\left\{\nu_{i}\right\}_{i=1}^{\infty}, \mu_{i} \in M_{i}, \nu_{i} \in N_{i}$, сходящиеся соответственно к точкам $\mu$ и $\nu$. Последовательность $\left\{\mu_{i}+\nu_{i}\right\}_{i=1}^{\infty}$ сходится к точке $\sigma$. Кроме того, для любого натурального $i$ точка $\mu_{i}+\nu_{i}$ принадлежит множеству $M_{i} \oplus N_{i}$ (по определению векторной суммы двух множеств). Таким образом, для точки $\sigma$ указана сходящаяся к ней последовательность точек, взятых по одной из множеств $M_{i} \oplus N_{i}$.

2. Пусть $\left\{\sigma_{i_{k}}\right\}_{k=1}^{\infty}-$ сходящаяся последовательность, $\sigma_{i_{k}} \in M_{i_{k}} \oplus N_{i_{k}}$, и $\lim _{k \rightarrow \infty} \sigma_{i_{k}}=\sigma$. Для каждой точки $\sigma_{i_{k}}$ верно соотношение $\sigma_{i_{k}}=\mu_{i_{k}}+\nu_{i_{k}}$, где $\mu_{i_{k}} \in M_{i_{k}}, \nu_{i_{k}} \in N_{i_{k}}$. Из последовательностей $\left\{\mu_{i_{k}}\right\}$ и $\left\{\nu_{i_{k}}\right\}$ можно поэтапно выбрать сходящиеся подпоследовательности $\left\{\mu_{j}\right\} \rightarrow \mu$ и $\left\{\nu_{j}\right\} \rightarrow \nu$, при этом $\sigma=\mu+\nu$. Поскольку $M_{i} \rightarrow M$ и $N_{i} \rightarrow N$, точки $\mu$ и $\nu$ принадлежат множествам $M$ и $N$ соответственно. Следовательно, $\sigma=\mu+\nu \in M \oplus N$. Лемма доказана.

ТЕОРемА 5.1 (о сумме Минковского однотипных примитивных параллелоэд-

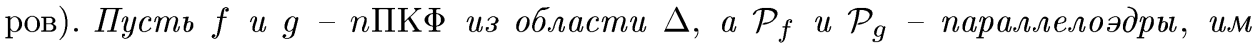
соответствующие. Пусть также $\mathcal{P}_{f+g}$ - параллелоэдр, соответствующий ПК $\Phi+g$. Тогда

$$
\mathcal{P}_{f+g}=\mathcal{P}_{f} \oplus \mathcal{P}_{g}
$$

ДокАЗАТЕЛЬСтво. 1. Напомним, что все параллелоэдры, соответствующие ПК $\Phi$ из области $\Delta$, имеют одинаковую структуру: параллелоэдр, отвечающий 
ПКФ с матрицей $W$ определен неравенствами

$$
2 x l_{i}^{T} \leqslant l_{i} W l_{i}^{T}, \quad i=\overline{1, s},
$$

кроме того, вершины параллелоэдров распадаются на классы когерентности.

2. Пусть $A$ и $B$ - матрицы ПКФ $f$ и $g$ соответственно. Отметим, что форма $f+g$, так же как и формы $f$ и $g$, принадлежит области $\Delta$, а ее матрицей является $A+B$.

Заметим, что $\mathcal{P}_{f} \oplus \mathcal{P}_{g} \subset \mathcal{P}_{f+g}$ : достаточно рассмотреть произвольные точки $\alpha_{0} \in \mathcal{P}_{f}$ и $\beta_{0} \in \mathcal{P}_{g}$ и сложить соответствуюшие неравенства (5.1). Получим

$$
2\left(\alpha_{0}+\beta_{0}\right) l_{i}^{T} \leqslant l_{i}(A+B) l_{i}^{T}, \quad i=\overline{1, s}
$$

т. е. точка $\alpha_{0}+\beta_{0}$ удовлетворяет всем неравенствам (5.1) для параллелоэдра $\mathcal{P}_{f+g}$.

3. Каждая вершина $\gamma_{0}$ параллелоэдра $\mathcal{P}_{f+g}$ принадлежит также и множеству $\mathcal{P}_{f} \oplus \mathcal{P}_{g}$. Действительно, пусть $l_{1}, l_{2}, \ldots, l_{n}$ - определяюшие $l$-векторы вершины $\gamma_{0}$, т. е. справедливы следуюшие соотношения:

$$
\begin{array}{ll}
2 \gamma_{0} l_{i}^{T}=l_{i}(A+B) l_{i}^{T}, & i=\overline{1, n} \\
2 \gamma_{0} l_{j}^{T} \leqslant l_{j}(A+B) l_{j}^{T}, & j=\overline{n+1, s}
\end{array}
$$

Отметим, что системе уравнений (5.2) не удовлетворяют никакие точки, кроме $\gamma_{0}$.

В параллелоэдрах $\mathcal{P}_{f}$ и $\mathcal{P}_{g}$ есть вершины, когерентные $\gamma_{0}$, т. е. существуют точки $\alpha_{0}$ и $\beta_{0}$, для которых

$$
\begin{array}{ll}
2 \alpha_{0} l_{i}^{T}=l_{i} A l_{i}^{T}, \quad i=\overline{1, n}, & 2 \alpha_{0} l_{j}^{T} \leqslant l_{j} A l_{j}^{T}, \quad j=\overline{n+1, s} \\
2 \beta_{0} l_{i}^{T}=l_{i} B l_{i}^{T}, \quad i=\overline{1, n}, \quad 2 \beta_{0} l_{j}^{T} \leqslant l_{j} B l_{j}^{T}, \quad j=\overline{n+1, s}
\end{array}
$$

соответственно. Сложением соответствующих соотношений (5.4) и (5.5) получаем, что для точки $\alpha_{0}+\beta_{0}$ выполнены условия (5.2) и (5.3). Следовательно, точки $\gamma_{0}$ и $\alpha_{0}+\beta_{0}$ совпадают (поскольку удовлетворяют системе $(5.2)$ ), в частности $\gamma_{0} \in$ $\mathcal{P}_{A} \oplus \mathcal{P}_{B}$

Таким образом, выпуклый многогранник $\mathcal{P}_{A} \oplus \mathcal{P}_{B}$ содержится в выпуклом многограннике $\mathcal{P}_{A+B}$ и притом содержит все его вершины. Это означает, что многогранники совпадают, т. е. $\mathcal{P}_{A+B}=\mathcal{P}_{A} \oplus \mathcal{P}_{B}$.

ТЕоремА 5.2 (краеугольная, о сумме Минковского реберных параллелоэдров, эквивалентная формулировка). Пусть $f \in \operatorname{clos} \Delta u$

$$
f=\sum_{i=1}^{w} \lambda_{i} \varphi_{i}, \quad \lambda_{i} \geqslant 0, \quad i=\overline{1, w}
$$

где $\varphi_{1}, \varphi_{2}, \ldots, \varphi_{w}$ - все реберные формы области $\Delta$. Пусть также $\mathcal{P}$ - параллелоэдр, соответствующий форме $f$, a $\mathcal{P}_{1}, \mathcal{P}_{2}, \ldots, \mathcal{P}_{w}-$ параллелоәдры, соответствующие формам $\varphi_{1}, \varphi_{2}, \ldots, \varphi_{w}$. Тогда

$$
\mathcal{P}=\lambda_{1} \mathcal{P}_{1} \oplus \lambda_{2} \mathcal{P}_{2} \oplus \cdots \oplus \lambda_{w} \mathcal{P}_{w}
$$


ДоКАЗАТЕЛЬСТВо. Не ограничивая общности рассуждений, мы можем считать, что $\sum_{i=1}^{w} \lambda_{i}=1$.

1. Сначала рассмотрим случай, когда $f$ - примитивная форма. Формы $\varphi_{i}$, вообше говоря, непримитивны. Рассмотрим последовательности ПКФ

$$
\left\{g_{i q}=\varphi_{i}+\frac{1}{q} f\right\}_{q=1}^{\infty}
$$

Кроме того, обозначим через $\mathcal{P}_{g_{i q}}$ параллелоэдр, соответствуюший ПКФ $g_{i q}$. Для любого натурального $q$ и $1 \leqslant i \leqslant w$ форма $g_{i q}$ принадлежит (открытой) области $\Delta$. Кроме того,

$$
\sum_{i=1}^{w} \lambda_{i} g_{i q}=\sum_{i=1}^{w} \lambda_{i}\left(\varphi_{i}+\frac{1}{q} f\right)=\sum_{i=1}^{w} \lambda_{i} \varphi_{i}+\frac{1}{q} \sum_{i=1}^{w} \lambda_{i} f=f+\frac{1}{q} f
$$

T. e.

$$
\sum_{i=1}^{w} \lambda_{i} g_{i q}=\left(1+\frac{1}{q}\right) f
$$

Поскольку и $f$, и все $g_{i q}-$ примитивные формы одного типа, согласно теореме 5.1 получаем

$$
\lambda_{1} \mathcal{P}_{g_{1 q}} \oplus \lambda_{2} \mathcal{P}_{g_{2 q}} \oplus \cdots \oplus \lambda_{w} \mathcal{P}_{g_{w q}}=\left(1+\frac{1}{q}\right) \mathcal{P} .
$$

Согласно теореме 4.1 имеем

$$
\lim _{q \rightarrow \infty} \mathcal{P}_{g_{i q}}=\mathcal{P}_{i}, \quad i=\overline{1, w} .
$$

Кроме того, очевидно, что

$$
\lim _{q \rightarrow \infty}\left(1+\frac{1}{q}\right) \mathcal{P}=\mathcal{P}
$$

Таким образом, из равенств (5.8), (5.9) и леммы 5.1 следует (5.6), т. е. утверждение теоремы для примитивной формы.

2. Пусть теперь $f$ - непримитивная форма. Зафиксируем произвольную примитивную форму $h \in \Delta$. Рассмотрим далее последовательности ПК $\Phi$

$$
\left\{g_{i q}=\varphi_{i}+\frac{1}{q} h\right\}_{i=1}^{\infty}
$$

Для любого натурального $q$ и $1 \leqslant i \leqslant w$ форма $g_{i q}$ принадлежит (открытой) области $\Delta$. Аналогично (5.7) получаем

$$
\sum_{i=1}^{w} \lambda_{i} g_{i q}=f+\frac{1}{q} h
$$

и затем

$$
\lambda_{1} \mathcal{P}_{g_{1 q}} \oplus \lambda_{2} \mathcal{P}_{g_{2 q}} \oplus \cdots \oplus \lambda_{w} \mathcal{P}_{g_{w q}}=\mathcal{P}_{f+\frac{1}{q} h} .
$$

Так же, как в п. 1 доказательства, справедливы равенства

$$
\lim _{q \rightarrow \infty} \mathcal{P}_{g_{i q}}=\mathcal{P}_{i}, \quad i=\overline{1, w} .
$$


Из теоремы 4.1 следует, что

$$
\lim _{q \rightarrow \infty} \mathcal{P}_{f+\frac{1}{q} h}=\mathcal{P}
$$

Из равенств (5.10) и (5.11), а также леммы 5.1 следует, что утверждение теоремы верно и для непримитивной формы $f \in \partial \Delta$.

Таким образом, соотношение (5.6) верно для любой формы из замкнутой области типа $\operatorname{clos} \Delta$. Теорема доказана.

\section{§6. Доказательство основной теоремы}

Приведем пример системы коренных параллелоэдров.

В каждой размерности $k$ выберем полный набор $\Delta_{k 1}, \Delta_{k 2}, \ldots, \Delta_{k, \sigma(k)}$ попарно неэквивалентных областей арифметических $L$-типов ПКФ. Обозначим через $\Sigma_{\Delta}$ объединение таких наборов для всех натуральных $k$.

Составим множество $\Sigma_{f}$ из всех реберных форм всех областей $\Delta_{k i} \in \Sigma_{\Delta}$. Обозначим через $\Sigma_{\mathcal{P}}$ множество всех параллелоэдров, соответствуюших квадратичным формам из множества $\Sigma_{f}$.

Из каждого класса попарно аффинно эквивалентных параллелоэдров, принадлежащих множеству $\Sigma_{\mathcal{P}}$, выберем по одному и только одному представителю. Множество всех таких представителей обозначим через $\Sigma^{\prime}$.

Быть может, в множестве $\Sigma^{\prime}$ найдутся параллелоэдры, являющиеся с точностью до аффиннитета взвешенной векторной суммой других параллелоэдров из этого множества. Удалим из множества $\Sigma^{\prime}$ все такие параллелоэдры и обозначим результат через $\Sigma$.

Мы утверждаем, что множество $\Sigma$ является системой коренных параллелоэдров.

Действительно, для системы $\Sigma$ первый пункт определения системы коренных параллелоэдров выполнен в силу того, что в каждой размерности $k$ сушествует только конечное число попарно неэквивалентных арифметических $L$-типов, в каждом из которых лиш конечное число реберных форм [1].

Второй пункт определения справедлив в силу краеугольной теоремы.

Справедливость третьего пункта обеспечивается указанной выше процедурой удаления лишних параллелоэдров из системы $\Sigma$. Основная теорема доказана.

Стоит отметить, что вопрос о том, существуют ли параллелоэдры, отвечающие реберным формам и являюшиеся притом суммами Минковского других параллелоэдров, открыт.

Мы также не знаем, существуют ли другие (кроме системы, состоящей из реберных параллелоэдров) системы коренных параллелоэдров.

\section{§7. Примеры}

Имея в виду, что в дальнейших публикациях мы собираемся рассмотреть примеры параллелоэдров высоких размерностей, ограничимся здесь размерностями $n=2$ и $n=3$, тем более, что параллелоэдры этих размерностей хорошо известны. 
При $n=2$ все арифметические $L$-типы попарно эквивалентны. Область каждого $L$-типа есть трехмерный трехгранный конус, попарно эквивалентным ребрам которого соответствуют параллелоэдры - отрезки. На рис. 2 изображена одна из таких областей: $0, c x_{1}^{2}, c x_{2}^{2}, c\left(x_{1}+x_{2}\right)^{2}$.

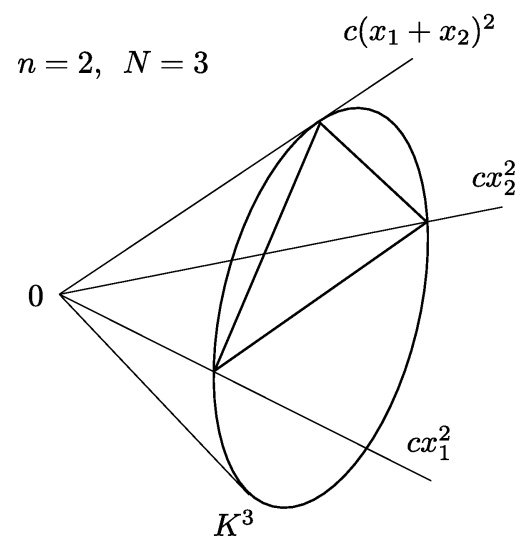

Рис. 2

Любой двумерный параллелоэдр является векторной суммой трех или двух отрезков в зависимости от того, лежит ли соответствующая квадратичная форма внутри области типа или на ее гранище (рис. 3).
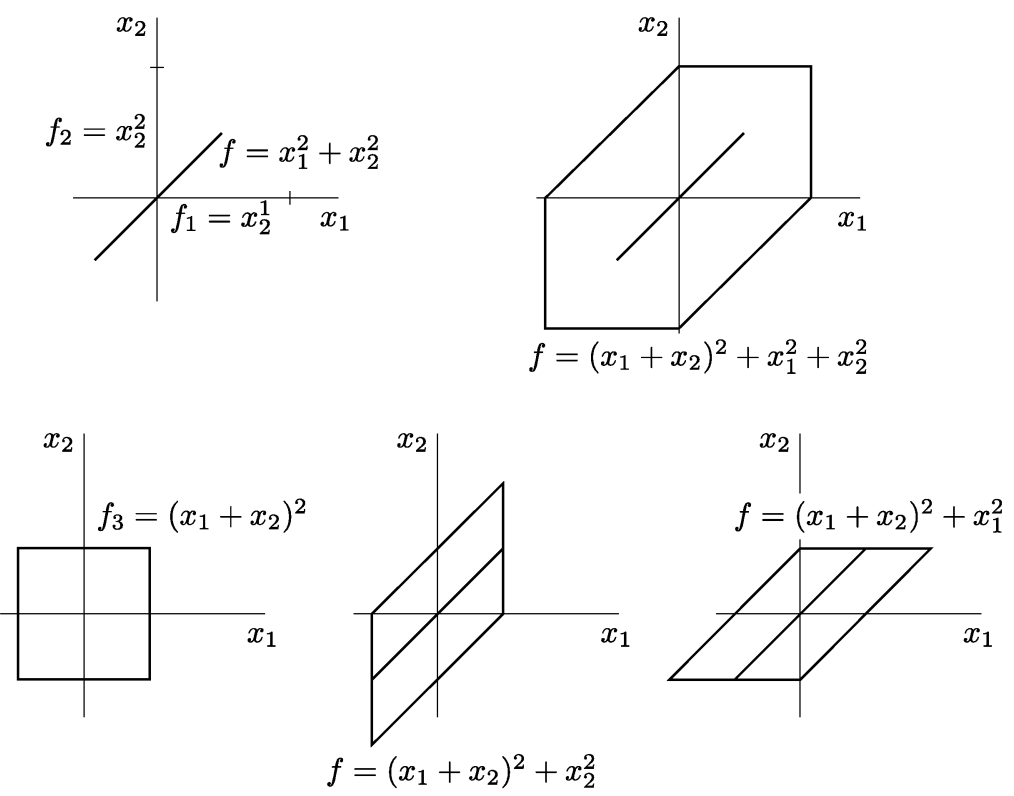

Рис. 3 
При $n=3$ все арифметические $L$-типы также попарно эквивалентны. Область типа есть шестимерный конус над пятимерным симплексом. Ребрам такого конуса также соответствуют параллелоэдры - отрезки. Трехмерные параллелоэдры представляют собой векторные суммы от трех до шести отрезков (рис. 4).

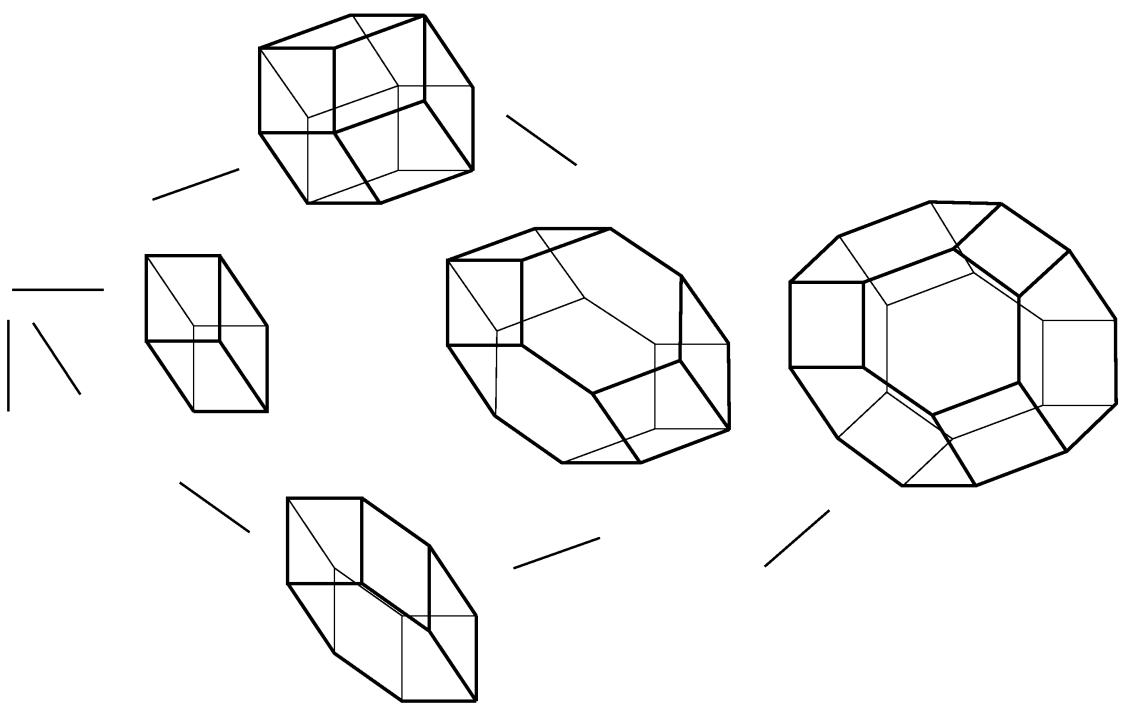

Рис. 4

Авторы выражают глубокую благодарность М. Д. Ковалёву и рецензенту за ценные советы и замечания, повлекшие улучшение текста статьи.

\section{Список литературы}

1. Вороной Г. Ф. Исследования о примитивных параллелоэдрах // Собр. соч. Т. 2. Киев: Изд-во АН УССР, 1952. С. 239-368 (оригинал см. J. reine und angew. Math. 1909. V. 136. P. 67-179).

2. Вороной Г. Ф. О некоторых свойствах положительных совершенных форм // Собр. соч. Т. 2. Киев: Изд-во АН УССР, 1952. С. 171-238 (оригинал см. J. reine und angew. Math. 1908. V. 133. P. 97-178).

3. Делоне Б. Н. Геометрия положительных квадратичных форм // УМН. 1937. №3. C. $16-62 ;$ 1938. № 4. C. 102-164.

4. Рьиков С. С., Барыкинский Р. Г., Кучериненко Я. В. Решение основных задач дискретной геометрии в случае плоскости. М.: Изд-во ЦПИ при мехмате МГУ, 2000. С. 1-96.

5. Рышков C. С., Барановский Е. П. Классические методы теории решетчатых упаковок // УМH. 1979. T. XXXIV. № 4(208). C. 3-63.

6. Michel L., Ryshkov S.S., Senechal M. An extension of Voronoii's theorem on primitive parallelotopes // Europ. J. Combinatorics. 1995. V. 16. P. 59-63.

7. Delaunay B. Sur la partition régulière l'espace a 4 dimensions // Изв. АН СССР. Отд. физ.-мат. наук. 1929. № 1. С. 79-110; № 2. С. 147-164.

8. Рьшков C. С. О структуре примитивного параллелоэдра и о последней проблеме Вороного // УМН. 1998. Т. 53. №2(320). С. 161-162.

9. Рьиков C.C. Прямое геометрическое описание произвольного $n$-мерного параллелоэдра второго типа Вороного // УМН. 1999. Т. 54. № 2. С. 18-19. 
10. Рышков C.C. О структуре примитивных параллелоэдров // Тез. докладов Международной школы-семинара по геометрии и анализу, посвященной 90-летию Н. В. Ефимова. Ростов-на-Дону, 2000. С. 63-65.

11. Рышков C.C. Коренные параллелоэдры и вершины областей типа $n$-мерных параллелоэдров // Материалы VII Международного семинара "Дискретная математика и ее приложения". М.: Изд-во ЦПИ при мехмате МГУ, 2001. С. 279-281.

12. Большакова E.A., Рьиков C.C. О неожиданно обретенной рукописи Г. Ф. Вороного // Чебышевский сборник. 2003. Т. 4. №3(7). С. 171-181.

13. Delaunay B. Sur la sphere vide // Proc. Internat. Congr. Math. Toronto: Univ. of Toronto Press, 1924. P. 695-700.

14. Большакова E.A. DV-разбиение и $L$-разбиение по Г.Ф. Вороному, Б.Н. Делоне и Б. А. Венкову // Чебышевский сборник. 2004. Т. 5. № 2(10). С. 30-41.

15. Рышков C.C., Барановский $E$. П. С-типы $n$-мерных решеток и пятимерные примитивные параллелоэдры (с приложением к теории покрытий) // Тр. МИАН СССР. 1976. T. 137. C. 1-132.

16. Венков Б.А. О проектировании параллелоэдров // Избр. труды. Л.: Наука, 1981. C. $362-379$.

Математический институт им. В. А. Стеклова РАН

Поступило в редакцию Московский государственный университет им. М. В. Ломоносова 